'Departamento de Gastroenterología. Facultad de Medicina. Pontificia Universidad Católica de Chile. Santiago de Chile.

${ }^{2}$ Unidad de Coloproctología. Departamento de Cirugía Digestiva. Facultad de Medicina. Pontificia Universidad Católica de Chile. Santiago de Chile. ${ }^{3}$ Departamento de Nutrición, Diabetes y Metabolismo. Facultad de Medicina. Pontificia Universidad Católica de Chile. Santiago de Chile.

Fuente de financiamiento: Este trabajo fue financiado por el Fondo Nacional de Ciencia y Tecnología de Chile (FONDECYT) de inicio $\mathrm{N}^{\circ} 11130502$ de C.H.-R. y FONDECYT regular $N^{\circ} 1131012$ de M.A.-L.

Recibido el 24 de agosto de 2015, aceptado el 29 de mayo de 2016 .

Correspondencia a: Dr. Cristian Hernández-Rocha Departamento de Gastroenterología. Pontificia Universidad Católica de Chile. Santiago de Chile. Marcoleta \# 367. Santiago de Chile.

Teléfono: +56223543820 . cristian.hernandez.rocha@gmail.com

\section{Diagnóstico y manejo de colitis ulcerosa grave. Una mirada actualizada}

\author{
CRISTIAN HERNÁNDEZ-ROCHA ${ }^{1}$, PATRICIO IBÁÑEZ ${ }^{1}$, \\ MARÍA ELENA MOLINA ${ }^{2}$, JULIETA KLAASSEN ${ }^{3}$, \\ ANDREA VALENZUELA ${ }^{3}$, ROBERTO CANDIA ${ }^{1}$, FELIPE BELLOLIO ${ }^{2}$, \\ ÁLVARO ZÚNIIGA ${ }^{2}$, RODRIGO MIGUIELES ${ }^{2}$, JUAN FRANCISCO MIQUEL ${ }^{1}$, \\ JOSÉ CHIANALE ${ }^{1}$, MANUEL ÁLVAREZ-LOBOS ${ }^{1}$
}

\section{Management of severe ulcerative colitis. An up-to-date}

Ulcerative Colitis (UC) is a chronic inflammatory disease involving the colon, with alternating periods of remission and activity. Exacerbations can be severe and associated with complications and mortality. Diagnosis of severe UC is based on clinical, biochemical and endoscopic variables. Patients with severe UC must be hospitalized. First line therapy is the use of intravenous corticoids which achieve clinical remission in most patients. However, 25\% of patients will be refractory to corticoids, situation that should be evaluated at the third day of therapy. In patients without response, cytomegalovirus infection must be quickly ruled out to escalate to second line therapy with biological drugs or cyclosporine. Total colectomy must not be delayed if there is no response to second line therapy, if there is a contraindication for second line therapies or there are complications such as: megacolon, perforation or massive bleeding. An active management with quick escalation on therapy allows to decrease the prolonged exposure to corticoids, reduce colectomy rates and its perioperative complications.

(Rev Med Chile 2017; 145: 96-105)

Key words: Colitis, Ulcerative; Colonic Diseases; Drug Therapy; Inflammatory Bowel Diseases.
$\mathrm{L}$ a enfermedad inflamatoria intestinal (EII) incluye 2 entidades: Colitis ulcerosa (CU) y la enfermedad de Crohn (EC). Ambas tienen un curso crónico con periodos de remisión y reactivación de variable intensidad. Los episodios de reactivación o crisis se caracterizan por la reaparición o aumento de la diarrea, sangre en deposiciones, dolor abdominal, con o sin síntomas sistémicos. En el caso de los pacientes con $\mathrm{CU}$, donde la afectación es exclusivamente del colon y recto, hasta $25 \%$ tendrá una crisis grave que requiere hospitalización, pudiendo darse esta situación al debut o en cualquier momento de la evolución ${ }^{1}$. De los pacientes con crisis grave, 20 a $30 \%$ no responderá al uso de corticoides intra- venosos y de ellos, $80 \%$ requerirá colectomía ${ }^{2}$. La mortalidad a corto plazo de la crisis grave es $\sim 1 \%{ }^{3}$. Por lo tanto, estos pacientes deben ser tratados oportuna y agresivamente, con el fin de evitar complicaciones y reducir la necesidad de colectomía.

La incidencia de CU y EC ha aumentado en los últimos años e importantes avances en el diagnóstico y manejo de estos pacientes han reducido la morbilidad y mortalidad ${ }^{4}$.

Lo señalado anteriormente determina que médicos de diversas áreas se vean enfrentados cada vez más frecuentemente a pacientes con CU grave. Por esto, el objetivo de este artículo es revisar y difundir el manejo actual de la crisis grave de CU. 


\section{Evaluación y manejo general de pacientes con crisis grave de colitis ulcerosa}

\section{Evaluación general e indices de gravedad}

La crisis grave de CU es una emergencia médica y su manejo se inicia con una evaluación clínica acuciosa que incluye consideraciones del estado general y hemodinámico del paciente, así como la identificación precoz de complicaciones como íleo, megacolon, perforación, hemorragia masiva o shock. En la Tabla 1 se señalan los exámenes mínimos que deben realizarse a todo paciente con crisis grave de CU, sin excluir la necesidad de otras evaluaciones según la condición clínica del paciente y el criterio del médico tratante.

Tabla 1. Exámenes mínimos a realizar en pacientes con crisis grave de colitis ulcerosa

\begin{tabular}{|l|}
\hline Hemograma completo y VHS \\
PCR \\
Creatinina \\
Electrolitos plasmáticos $\left(\mathrm{Na}^{+}, \mathrm{K}^{+}, \mathrm{Cl}^{-}, \mathrm{Mg}^{++}\right)$ \\
Albúmina y prealbúmina \\
Glicemia \\
Pruebas hepáticas \\
Estudio de Clostridium difficile en deposiciones \\
\hline
\end{tabular}

VHS: velocidad de eritrosedimentación; PCR: proteína C reactiva.
La crisis grave de CU se define por los criterios establecidos por Truelove y Witts ${ }^{5}$ y son aquellos pacientes que presentan 6 o más deposiciones diarreicas por día, con o sin sangre y asociados a cualquiera de los siguientes síntomas o signos de toxicidad sistémica: fiebre $\left(\mathrm{T}^{\circ}>37,8^{\circ} \mathrm{C}\right)$, taquicardia (frecuencia cardiaca $\geq 90$ latidos/min), anemia (hemoglobina $<10,5 \mathrm{~g} / \mathrm{dl}$ ), velocidad de eritrosedimentación elevada (VHS $\geq 30 \mathrm{~mm} /$ hora). Estas variables son fácilmente evaluables ambulatoriamente y permiten tomar conductas para el manejo de estos pacientes.

Para evaluar más objetivamente la actividad de la CU también existen índices de gravedad, siendo los más utilizados en estudios y en la práctica clínica los de Truelove y Witts modificados ${ }^{1}$, que sólo incluye variables clínicas y de laboratorio (Tabla 2) y los de la Clínica Mayo o $\mathrm{DAI}^{6}$ (por sus siglas en inglés, Disease Activity Index), que incluyen variables clínicas y endoscópicas (Tabla 3 ).

Si bien la aplicación de alguno de estos índices no es imprescindible, son de utilidad para categorizar en forma más objetiva la gravedad del paciente y para el seguimiento.

Los pacientes con crisis grave deben ser hospitalizados y manejados desde su ingreso por un equipo multidisciplinario, constituido por gastroenterólogos, nutriólogos y coloproctólogos (o en su defecto por cirujanos con experiencia en cirugía de colon).

Una vez hospitalizados, la reposición de vo-

Tabla 2. Índice de gravedad de Truelove-Witts modificado

\begin{tabular}{|llll|}
\hline & $\mathbf{1}$ punto & $\mathbf{2}$ puntos & 3 puntos \\
\hline $\mathrm{n}$ deposiciones/día & $<4$ & 4 a 6 & $>6$ \\
\hline Sangre en deposiciones & - & + & $++/+++$ \\
\hline Temperatura axilar $\left({ }^{\circ} \mathrm{C}\right)$ & $<37$ & $37-38$ & $>38$ \\
Frecuencia cardiaca & $<80$ & 80 a 100 & $>100$ \\
Hemoglobina $(\mathrm{g} / \mathrm{dl})$ & $>14$ & & $<10$ \\
$\quad-$ Hombres & $>12$ & 14 a 10 & $<10$ \\
$\quad-$ Mujeres & $<10.000$ & 12 a 10 & $>13.000$ \\
Leucocitos/ul & $<15$ & 10.000 a 13.000 & $>30$ \\
VHS (mm/h) & $>3,2$ & 15 a 30 & $<3,0$ \\
Albuminemia $(\mathrm{g} / \mathrm{dl})$ & $>3,8$ & 3,2 a 3,0 & $<3$ \\
\hline Potasio $(\mathrm{meq} / \mathrm{l})$ & 3,8 a 3 & \\
\hline
\end{tabular}

Inactivo: Menos de 11 puntos; Leve: 11 a 15 puntos; Moderado: 16 a 21 puntos; Grave: 22 a 27 puntos. VHS: velocidad de eritrosedimentación. 
Tabla 3. Índice de gravedad de la Clínica Mayo

\begin{tabular}{|lllll|}
\hline & O punto & $\mathbf{1}$ punto & $\mathbf{2}$ puntos & 3 puntos \\
$\begin{array}{l}\text { Frecuencia de } \\
\text { deposiciones }\end{array}$ & $\begin{array}{l}\text { Normal (lo habitual } \\
\text { para el paciente) }\end{array}$ & $\begin{array}{l}\text { 1 a } 2 \text { deposiciones } \\
\text { > a lo normal }\end{array}$ & $\begin{array}{l}3 \text { a } 4 \text { deposiciones } \\
\text { > a lo normal }\end{array}$ & $\begin{array}{l}\text { > a } 4 \text { deposiciones } \\
\text { sobre lo normal }\end{array}$ \\
Sangrado & Sin sangrado & $\begin{array}{l}\text { Sangrado visible en } \\
\text { menos de } 50 \% \text { de las } \\
\text { deposiciones }\end{array}$ & $\begin{array}{l}\text { Sangrado visible en } \\
50 \% \text { o más de las } \\
\text { deposiciones }\end{array}$ & Sólo sangre \\
$\begin{array}{l}\text { Apariencia endoscópica } \\
\text { de la mucosa }\end{array}$ & $\begin{array}{l}\text { Normal o enfermedad } \\
\text { inactiva }\end{array}$ & $\begin{array}{l}\text { Eritema, disminución } \\
\text { patrón vascular y } \\
\text { friabilidad leve }\end{array}$ & $\begin{array}{l}\text { Eritema marcado, } \\
\text { ausencia de patrón } \\
\text { vascular, friabilidad y } \\
\text { erosiones }\end{array}$ & $\begin{array}{l}\text { Sangrado úlceras } \\
\text { espontáneo o }\end{array}$ \\
Evaluación global & Normal & Leve & Moderado & Grave \\
\hline
\end{tabular}

Remisión: $\leq 2$ puntos sin ningún subscore > 1; Leve: 3 a 4 puntos; Moderada: 5 a 8 puntos; Grave: 9 a 12 puntos.

lumen con cristaloides es importante y debe ser titulada de acuerdo a variables hemodinámicas como presión arterial, frecuencia cardiaca y diuresis. En pacientes con anemia, esta puede ser tratada con transfusión de glóbulos rojos o aporte de hierro intravenoso de acuerdo a variables hemodinámicas y comorbilidades del paciente. El objetivo debe ser mantener una hemoglobina entre 8 y $10 \mathrm{~g} / \mathrm{dl}^{7}$.

Debe evitarse el uso de antiinflamatorios no esteroidales ya que estos pueden exacerbar la $\mathrm{CU}^{8,9}$. La fiebre debería tratarse con medidas físicas y paracetamol.

Hipokalemia e hipomagnesemia podrían desencadenar megacolon por lo que deben ser corregidas $^{10,11}$.

Es reconocido que pacientes con EII activa se encuentran en un estado de hipercoagulabilidad, por lo tanto, la administración de heparina profiláctica subcutánea está indicada para prevenir eventos tromboembólicos ${ }^{12}$. El uso de heparina es seguro en estos pacientes y la presencia de sangre en deposiciones no es un impedimento para su uso, a menos que exista una hemorragia hemodinámicamente significativa ${ }^{13}$.

Fármacos como anticolinérgicos y narcóticos pueden precipitar la aparición de megacolon, por lo que debe evitarse su uso ${ }^{10}$. En el pequeño grupo de pacientes en que el dolor es un problema, el uso juicioso de opioides puede ser aceptable, con monitorización de signos de megacolon y síndrome de intestino narcótico ${ }^{14}$. El uso de antidiarreicos está contraindicado, dado el riesgo de desarrollo de megacolon.

\section{Infección por Clostridium difficile y otros enteropatógenos en CU grave}

La frecuencia de la infección por $C$. difficile (ICD) en pacientes con EII se ha incrementa$\mathrm{do}^{15}$. Además, los pacientes con ICD y EII tienen estadías hospitalarias más prolongadas, mayor tasa de colectomía y mortalidad que aquellos con solo EII ${ }^{16}$.

Si bien los factores de riesgo para ICD son similares a la población general, $C$. difficle puede gatillar crisis de CU sin la presencia de estos factores, incluso en ausencia de uso previo de antibióticos ${ }^{17}$. Por lo tanto, todo paciente con exacerbación de CU debe investigarse ICD, independiente de sus antecedentes. Los estudios microbiológicos de elección, por su alta sensibilidad, son aquellos basados en técnicas de amplificación de ácidos nucleicos en deposiciones ${ }^{18}$.

En pacientes con EII, estudios han mostrado que el tratamiento con metronidazol oral de la ICD se asocia a mayores tasas de falla de tratamiento y recurrencia, comparado con vancomicina oral ${ }^{19}$. Por lo tanto, se recomienda el uso de esta última como terapia de primera línea en crisis grave de CU asociada a ICD. Una vez que se inicia el tratamiento adecuado de la ICD, los tratamientos de la crisis grave de $\mathrm{CU}$, como corticoides, se pueden mantener.

Con respecto a otras infecciones entéricas bacterianas o parasitarias, no existe claridad si deben buscarse en pacientes con crisis de CU. Infecciones por Campylobacter jejuni han sido encontradas frecuentemente en pacientes con crisis de $\mathrm{CU}^{20}$. 
Por otro lado, infecciones parasitarias, como las producidas por Entamoeba histolytica, son encontradas infrecuentemente en países desarrollados ${ }^{21}$, no obstante en países latinoamericanos aún se encuentran altas tasas de parasitosis, las cuales son mayores en pacientes con $\mathrm{EII}^{22}$. Por lo tanto, colitis infecciosas deben ser excluidas con coprocultivo y eventualmente parasitológico seriado de deposiciones, sobre todo ante el inicio abrupto de diarrea, vómitos o fiebre alta, antecedentes de contactos o viajes. Sin embargo, el tratamiento no debe ser retrasado mientras se esperan los resultados microbiológicos.

El tratamiento antibiótico empírico no ha demostrado beneficio en pacientes con CU gra$\mathrm{ve}^{23-25}$. Situaciones en que se considera el uso de antibióticos son: antecedentes epidemiológicos de exposición (viajes, contactos afectados); infección demostrada por un enteropatógeno, signos de sepsis o megacolon tóxico.

\section{Rol de citomegalovirus en crisis grave de enfermedad inflamatoria intestinal}

El citomegalovirus (CMV) es un virus $\mathrm{ADN}$ perteneciente a la familia Herpesviridae y como tal produce una infección latente crónica que afecta a 30 a $90 \%$ de los adultos en distintas series ${ }^{26-28}$. Sus principales reservorios son el endotelio y los monocito-macrófagos. El sistema inmune adaptativo permite que la infección sea asintomática, evitando la replicación y la reactivación de $\mathrm{CMV}^{29-}$ ${ }^{31}$. La EII es una condición de riesgo de reactivación viral, tanto por la inmunosupresión como por los episodios de crisis, en donde la liberación de citoquinas y el reclutamiento de monocitos-macrófagos favorecen la replicación local de $\mathrm{CMV}^{31-33}$.

En la CU, la reactivación local de CMV se manifiesta habitualmente por crisis graves refractarias a corticoides ${ }^{31,32,34-37}$. Estudios de cohorte informan una prevalencia de reactivación viral que varía entre 4,6 y $25 \%{ }^{38-44}$.

El diagnóstico se puede realizar mediante técnicas de detección en sangre periférica y muestras tisulares. La antigenemia de CMV es la alternativa en sangre periférica más utilizada, corresponde a la detección de la proteína viral pp65 en leucocitos periféricos ${ }^{45}$. La serología para CMV no se recomienda, dada su baja sensibilidad y difícil interpretación ${ }^{46-48}$. La detección tisular es superior a las técnicas en sangre, ya que permite buscar evidencia de replicación local en biopsias de colon, ya sea mediante tinción con hematoxilina-eosina (sensibilidad 30-50\%), inmunohistoquímica (IHQ, sensibilidad 60-90\%) o reacción de polimerasa en cadena (RPC, sensibilidad mayor a $90 \%)^{29,31,49}$. En las publicaciones más recientes, las técnicas más utilizadas son inmunohistoquímica o RPC en biopsia de mucosa colónica, dada su mayor sensibilidad ${ }^{38-43}$.

Nuestra recomendación es buscar dirigidamente la reactivación de CMV en crisis graves cortico-refractarias al tercer día de tratamiento a través de IHQ o RCP en biopsias obtenidas en la rectosigmoidoscopía ${ }^{50}$. En pacientes con estudio positivo, la medición de carga viral en sangre o antigenemia pueden ayudar a guiar la terapia, aunque los valores de corte no son claros. La terapia antiviral sugerida es ganciclovir endovenoso por 14 días, cuya tasa de respuesta varía entre 60 y $86 \%$; sin embargo, la evidencia está limitada a estudios observacionales de tipo cohorte ch,45,49,51-53. $^{\text {. }}$ La respuesta se observa al quinto o séptimo día de tratamiento.

\section{Estudios de imágenes y endoscópicos en CU grave}

Pacientes con crisis grave de $\mathrm{CU}$ deben ser evaluados con radiografía de abdomen simple a su ingreso, con el fin de detectar precozmente signos de íleo, megacolon o perforación. El hallazgo de íleo o dilatación del colon (diámetro de colon transverso $\geq 5,5 \mathrm{~cm}$ ) se asocia a mayores tasas de colectomía ${ }^{54,55}$. Además, en algunos casos permite estimar la extensión de la enfermedad y realizar una evaluación basal para comparar en caso de deterioro clínico ${ }^{56}$. Evaluaciones con tomografía axial computarizada (TAC) o resonancia magnética (RM) de abdomen deben ser reservados para pacientes con sospecha de complicaciones.

La rectosigmoidoscopía flexible con biopsia de la mucosa afectada es parte de la evaluación de los pacientes con debut o exacerbación de CU. En los primeros permite confirmar el diagnóstico y evaluar la gravedad de la enfermedad ${ }^{57}$, mientras que en los pacientes con exacerbación permite evaluar la gravedad y tomar muestras para búsqueda de CMV. La rectosigmoidoscopía sin preparación y con mínima insuflación es segura 
en manos experimentadas ${ }^{58}$. La colonoscopía total, si bien ha sido reportada segura en pacientes con crisis grave, se puede asociar a mayor tasa de complicaciones, tanto por el procedimiento como por la preparación. Dado que no aporta mayores datos que la rectosigmoidoscopía, en el escenario de CU grave la colonoscopía total no es recomendada ${ }^{59}$.

\section{Evaluación y manejo nutricional}

El soporte nutricional es parte fundamental del tratamiento de la crisis grave de CU. Existen varios mecanismos fisiopatológicos que explican la alta prevalencia de compromiso nutricional $(75 \%)^{60}$ : disminución de ingesta (anorexia, dolor abdominal, restricciones dietéticas), aumento de requerimientos calóricos-proteicos (inflamación, complicaciones infecciosas y/o tratamiento esteroidal) o malabsorción ${ }^{60,61}$.

$\mathrm{Al}$ ingreso debe realizarse evaluación nutricional con cuantificación de ingesta calórico-proteica, antropometría que incluye peso, talla, índice de masa corporal (IMC), pliegue tricipital y circunferencia muscular braquial. Otras variables a considerar son porcentaje de pérdida de peso, dinamometría y calorimetría indirecta, si es posible, para ajustar requerimientos calóricos. Esta evaluación, más la determinación de prealbúmina, albúmina, proteína $\mathrm{C}$ reactiva (PCR) y nitrógeno urinario total, permiten diagnosticar y estimar la magnitud, tanto de la desnutrición como del catabolismo, para ajustar el plan de soporte nutricional y monitorizar su eficacia ${ }^{60-62}$.

A menos que esté contraindicada, la vía enteral es la de elección. $\mathrm{Al}$ ingreso se indica régimen de ayuno por $24 \mathrm{~h}$ y, según evolución clínica, se progresa desde hídrico a régimen sin residuos, utilizando suplementos orales $(600 \mathrm{Kcal} /$ día en adición a la dieta). Si hay intolerancia, se inicia nutrición enteral continua con formula polimérica. Si no es posible la instalación de sonda enteral o estuviera contraindicada se inicia nutrición parenteral ${ }^{63}$.

Los pacientes candidatos a cirugía con albúmina sérica $<$ a $3 \mathrm{~g} / \mathrm{l}$ o pérdida de peso significativa $(>10 \%$ los últimos 3 a 6 meses o IMC $<18,5 \mathrm{~kg} /$ $\left.\mathrm{m}^{2}\right)$ tienen más complicaciones postoperatorias, por lo que deben recibir soporte nutricional preoperatorio, de preferencia por vía enteral. En estos pacientes con frecuencia el apoyo parenteral es necesario para alcanzar balance energético y nitrogenado positivo. Se requiere un mínimo de 5 días de soporte nutricional preoperatorio para disminuir las complicaciones postoperatorias. $\mathrm{Si}$ la cirugía no es urgente, es preferible apoyar a los pacientes hasta que la actividad de la enfermedad sea menor y presenten una albúmina por encima de $3 \mathrm{~g} / \mathrm{l}$. Independiente de la vía de soporte nutricional, deben reevaluarse parámetros nutricionales al quinto o sexto día de soporte para ajustar los aportes ${ }^{59,63-66}$.

Dado que los pacientes con EII presentan una alta prevalencia de déficit de micronutrientes, siendo más frecuente en EC que en CU, se sugiere identificar el déficit de micronutrientes como vitamina $\mathrm{D}$, ácido fólico, vitamina $\mathrm{B} 12$ y fierro. Suplementar en caso de ser necesario incluyendo además calcio y zinc ${ }^{61}$.

\section{Tratamiento}

\section{Tratamiento de primera línea}

Los corticoides siguen siendo la terapia de primera línea en la crisis grave de $\mathrm{CU}$, ya que reducen la morbilidad y mortalidad ${ }^{3,5,67}$. El uso de dosis mayores a $60 \mathrm{mg}$ de metilprednisolona o equivalente no es recomendado dada la falta de evidencia de mayor eficacia y el aumento de efectos adversos, no existiendo diferencias tampoco entre los diferentes corticoides o administración en bolo o infusión ${ }^{3,68,69}$. Por esto, se recomienda la administración de metilprednisolona $20 \mathrm{mg}$ cada 8 h o hidrocortisona $100 \mathrm{mg}$ cada 8 h endovenosos.

\section{Evaluación al tercer día (cortico-refractariedad)}

Si el paciente no ha respondido a la terapia inicial con corticoides endovenosos, se considera cortico-refractario y candidato a terapias de segunda línea, cuyo objetivo es evitar la colectomía de urgencia e idealmente lograr una remisión que pueda ser mantenida a largo plazo.

Tradicionalmente se ha considerado una crisis grave de CU como cortico-refractaria cuando no responde a tratamiento con corticoides endovenosos luego de 7 días. Sin embargo, este plazo se ha acortado a 3 días en los últimos años, para evitar la exposición prolongada a corticoides en pacientes potenciales candidatos a colectomía. Así, se han utilizado índices para evaluar precozmente la cortico-refractariedad en el escenario 
de CU grave. El más utilizado es el de Oxford, que evalúa al tercer día de tratamiento con corticoides la frecuencia de deposiciones y la PCR ${ }^{55}$. Pacientes con una frecuencia de deposiciones mayor a 8 por día o frecuencia de deposiciones de 3 a 8 por día asociado a una PCR igual o mayor de $45 \mathrm{mg} / \mathrm{L}$ son considerados refractarios y con un alto riesgo de colectomía. En estos pacientes debe evaluarse la reactivación de CMV e iniciarse terapias de segunda línea. Además, se debe evaluar la presencia de infección por virus de la hepatitis B y C, serología para virus de inmunodeficiencia adquirida (VIH) y tuberculosis latente (radiografía de tórax asociado a test de tuberculina o ensayos de liberación de interferón gamma) antes del inicio de terapia inmunosupresora más potente. Si la obtención de los resultados de estos exámenes es lenta, se sugiere realizarlos al ingreso del paciente con crisis grave de CU.

\section{Tratamiento de segunda línea}

Las opciones en pacientes cortico-refractarios son ciclosporina o terapias biológicas como infliximab. Estos fármacos deberían ser manejados en centros con experiencia en su uso. En aquellos pacientes que no han recibido terapia previa con inmunomoduladores del tipo tiopurínicos (azatioprina o 6-mercaptopurina), la ciclosporina es una buena opción y se recomienda iniciar con dosis de $2 \mathrm{mg} / \mathrm{kg} /$ día endovenoso y ajustarla según niveles plasmáticos, logrando concentraciones de 100-200 ng/ml por 7 días y luego cambiar a terapia oral en dosis de $5 \mathrm{mg} / \mathrm{kg} /$ día por un mes. Si existe una adecuada respuesta se puede continuar terapia con tiopurínicos. En aquellos pacientes que han recibido tratamiento con tiopurínicos, la opción es el uso de infliximab en inducción con $5 \mathrm{mg} / \mathrm{kg}$ a las 0,2 y 6 semanas. Recientemente se demostró que ambas terapias son igualmente efectivas en evitar la colectomía, por lo tanto, la decisión debe basarse en la experiencia y recursos del centro, así como en las comorbilidades y preferencias del paciente ${ }^{70}$. La respuesta a ambas terapias se observa al quinto a séptimo día de tratamiento. En pacientes con triple inmunosupresión o en tratamiento con ciclosporina, se sugiere profilaxis contra Peumocystis jirovesii con cotrimoxazol oral.

En caso de fracaso de una de las terapias de segunda línea, algunas series reportan el uso de ciclosporina después de infliximab o viceversa; sin embargo, la eficacia de esta estrategia mues- tra resultados variables y existe un alto riesgo de efectos secundarios, principalmente infecciones. Su aplicación se recomienda sólo en centros con alta experiencia en el manejo de pacientes con crisis grave de $\mathrm{EII}^{71,72}$.

De esta forma, ante el fracaso a terapias de segunda línea o si existe contraindicación a su uso, la cirugía realizada por cirujanos con experiencia es la opción para la resolución de la enfermedad.

\section{Manejo quirúrgico}

La causa más frecuente de cirugía de urgencia por crisis grave de CU está constituida por aquellos pacientes que no tienen respuesta al tratamiento médico intensivo. Otras causas de cirugía de urgencia mucho menos frecuentes son el megacolon tóxico, la perforación del colon y la hemorragia masiva.

Tomar la decisión de realizar la cirugía precozmente puede llevarnos a operar un mayor número de pacientes y, por otro lado, la demora en tomar la decisión quirúrgica puede conducir a que operemos pacientes en peores condiciones generales, más desnutridos e inmunodeprimidos, lo que conlleva un aumento de la morbilidad y mortalidad postoperatoria ${ }^{73}$. Por lo tanto, la eficacia de la terapia médica debe ser evaluada precozmente, como se señaló en secciones previas. El paciente y su familia deben ser informados del pronóstico y de las alternativas terapéuticas, tanto médicas como quirúrgicas, desde su ingreso por crisis grave de CU.

Una vez que se ha tomado la decisión de someter al paciente a una cirugía de urgencia, la colectomía total con ileostomía terminal es el procedimiento de elección ${ }^{74,75}$. De esta forma, se evita la morbilidad de la disección pélvica y no se realiza una anastomosis intestinal que potencialmente podría filtrar. Por otro lado, mantiene abiertas todas las posibilidades de reconstrucción futura del tránsito intestinal. La mortalidad de la cirugía es $<1 \%$ en centros especializados y la morbilidad fluctúa entre 33 y $42 \%{ }^{74}$.

En la actualidad, el abordaje mínimamente invasivo (laparoscópico), realizado por cirujanos entrenados en centros con experiencia, es seguro y tiene resultados comparables a la cirugía abierta en términos de morbimortalidad postoperatoria. Más aun, se ha reportado una menor tasa de 
complicaciones en los pacientes operados por vía laparoscópica vs abierta $(20 \% \text { vs } 57 \%)^{76}$. El contar con enfermeras especialistas en el manejo de ostomas ayuda en el manejo a corto y largo plazo de los pacientes con ileostomía.

\section{Conclusión}

La crisis grave de CU es una emergencia médica que como tal requiere un manejo oportuno y agresivo. Corticoides sistémicos y nuevas terapias han disminuido la morbilidad y mortalidad de este cuadro. El manejo multidisciplinario con escalamiento rápido en la terapia médica evitando el uso prolongado de corticoides constituye el paradigma de manejo actual. La colectomía sigue siendo la terapia de rescate en pacientes que no responden a terapia médica y su implementación no debe ser retrasada innecesariamente. Según lo revisado en este artículo proponemos un algoritmo de manejo de la crisis grave de CU, el cual se presenta en la Figura 1.

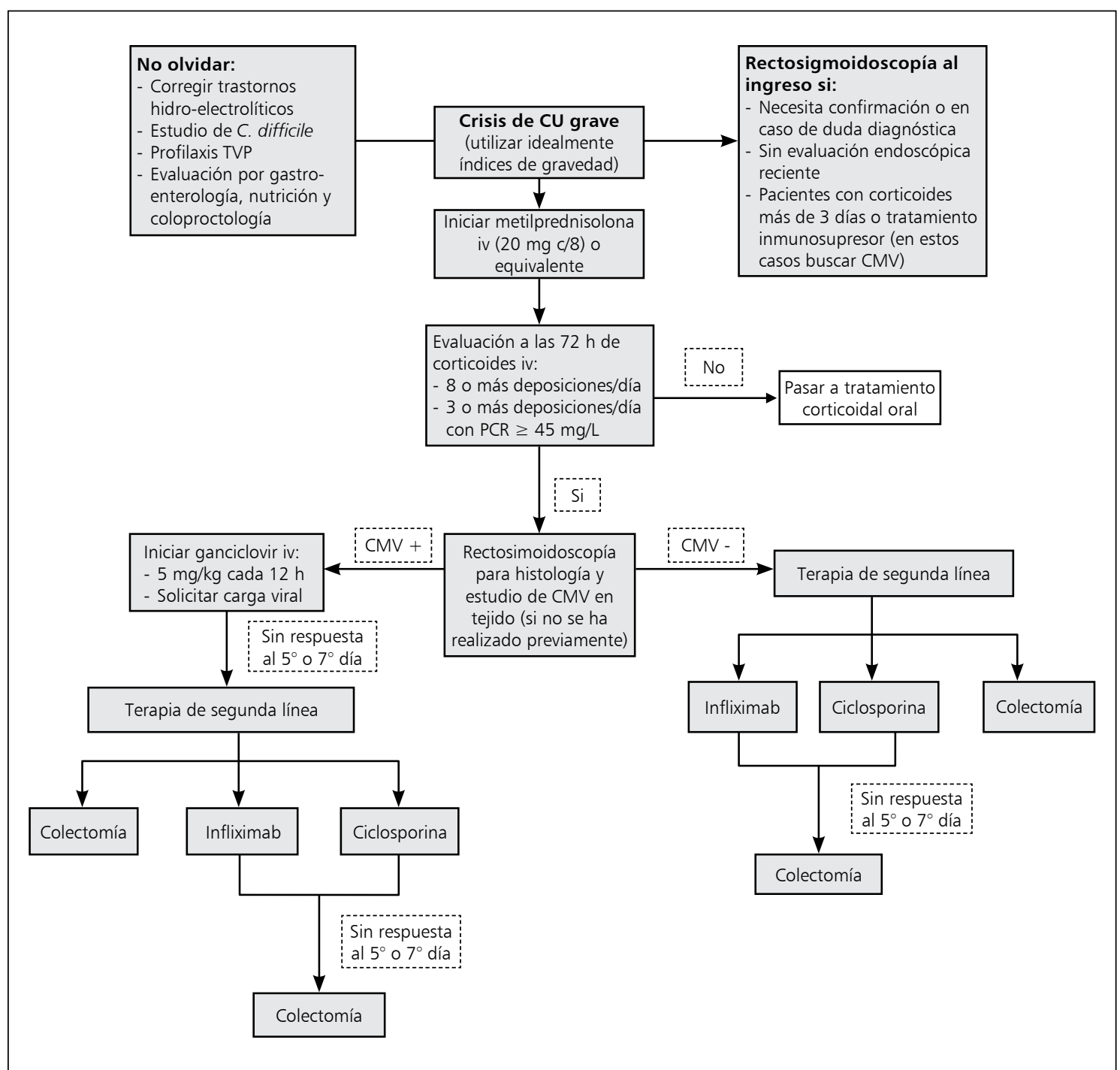

Figura 1. Algoritmo de manejo de la crisis grave de colitis ulcerosa. CU: colitis ulcerosa. TVP: trombosis venosa profunda. CMV: citomegalovirus. IV: intravenoso. PCR: proteína C reactiva. 


\section{Referencias}

1. Dinesen LC, Walsh AJ, Protic MN, Heap G, Cummings F, Warren BF, et al. The pattern and outcome of acute severe colitis. J Crohns Colitis 2010; 4 (4): 431-7.

2. Aratari A, Papi C, Clemente V, Moretti A, Luchetti R, Koch $\mathrm{M}$, et al. Colectomy rate in acute severe ulcerative colitis in the infliximab era. Dig Liver Dis 2008; 40 (10): 821-6.

3. Turner D, Walsh CM, Steinhart AH, Griffiths AM. Response to corticosteroids in severe ulcerative colitis: a systematic review of the literature and a meta-regression. Clin Gastroenterol Hepatol 2007; 5 (1): 103-10.

4. Cosnes J, Gower-Rousseau C, Seksik P, Cortot A. Epidemiology and natural history of inflammatory bowel diseases. Gastroenterology 2011; 140 (6): 1785-94.

5. Truelove SC, Witts LJ. Cortisone in ulcerative colitis; final report on a therapeutic trial. Br Med J 1955; 2 (4947): 1041-8.

6. Schroeder KW, Tremaine WJ, Ilstrup DM. Coated oral 5-aminosalicylic acid therapy for mildly to moderately active ulcerative colitis. A randomized study. N Engl J Med 1987; 317 (26): 1625-9.

7. García-Erce JA, Gomollon F, Muñoz M. Blood transfusion for the treatment of acute anaemia in inflammatory bowel disease and other digestive diseases. World J Gastroenterol 2009; 15 (37): 4686-94.

8. Felder JB, Korelitz BI, Rajapakse R, Schwarz S, Horatagis AP, Gleim G. Effects of nonsteroidal antiinflammatory drugs on inflammatory bowel disease: a case-control study. Am J Gastroenterol 2000; 95 (8): 1949-54.

9. Kaufmann HJ, Taubin HL. Nonsteroidal anti-inflammatory drugs activate quiescent inflammatory bowel disease. Ann Intern Med 1987; 107 (4): 513-6.

10. Gan SI, Beck PL. A new look at toxic megacolon: an update and review of incidence, etiology, pathogenesis, and management. Am J Gastroenterol 2003; 98 (11): 2363-71.

11. Cohn EM, Copit P, Tumen HJ. Ulcerative colitis with hypopotassemia. Gastroenterology 1956; 30 (6): 950-7.

12. Yuhara H, Steinmaus C, Corley D, Koike J, Igarashi M, Suzuki T, et al. Meta-analysis: the risk of venous thromboembolism in patients with inflammatory bowel disease. Aliment Pharmacol Ther 2013; 37 (10): 953-62.

13. Shen J, Ran ZH, Tong JL, Xiao SD. Meta-analysis: The utility and safety of heparin in the treatment of active ulcerative colitis. Aliment Pharmacol Ther 2007; 26 (5): 653-63.

14. Grunkemeier DM, Cassara JE, Dalton CB, Drossman DA. The narcotic bowel syndrome: clinical features, pathophysiology, and management. Clin Gastroenterol Hepatol 2007; 5 (10): 1126-39; quiz 1-2.
15. Rodemann JF, Dubberke ER, Reske KA, Seo da H, Stone $\mathrm{CD}$. Incidence of Clostridium difficile infection in inflammatory bowel disease. Clin Gastroenterol Hepatol 2007; 5 (3): 339-44.

16. Ananthakrishnan AN, McGinley EL, Binion DG. Excess hospitalisation burden associated with Clostridium difficile in patients with inflammatory bowel disease. Gut 2008; 57 (2): 205-10.

17. Mylonaki M, Langmead L, Pantes A, Johnson F, Rampton DS. Enteric infection in relapse of inflammatory bowel disease: importance of microbiological examination of stool. Eur J Gastroenterol Hepatol 2004; 16 (8): 775-8.

18. Hernández-Rocha C, Barra-Carrasco J, Álvarez-Lobos M, Paredes-Sabja D, Guzmán-Durán AM. Prospective comparison of a commercial multiplex real-time polymerase chain reaction and an enzyme immunoassay with toxigenic culture in the diagnosis of Clostridium difficile-associated infections. Diagn Microbiol Infect Dis 2013; 75 (4): 361-5.

19. Horton HA, Dezfoli S, Berel D, Hirsch J, Ippoliti A, McGovern D, et al. Antibiotics for Treatment of Clostridium difficile Infection in Hospitalized Patients with Inflammatory Bowel Disease. Antimicrob Agents Chemother 2014; 58 (9): 5054-9.

20. Navarro-Llavat M, Domenech E, Bernal I, Sanchez-Delgado J, Manterola JM, Garcia-Planella E, et al. Prospective, observational, cross-sectional study of intestinal infections among acutely active inflammatory bowel disease patients. Digestion 2009; 80 (1): 25-9.

21. Meyer AM, Ramzan NN, Loftus EV, Jr., Heigh RI, Leighton JA. The diagnostic yield of stool pathogen studies during relapses of inflammatory bowel disease. J Clin Gastroenterol 2004; 38 (9): 772-5.

22. Yamamoto-Furusho JK, Torijano-Carrera E. Intestinal protozoa infections among patients with ulcerative colitis: prevalence and impact on clinical disease course. Digestion 2010; 82 (1): 18-23.

23. Chapman RW, Selby WS, Jewell DP. Controlled trial of intravenous metronidazole as an adjunct to corticosteroids in severe ulcerative colitis. Gut 1986; 27 (10): 1210-2.

24. Mantzaris GJ, Hatzis A, Kontogiannis P, Triadaphyllou G. Intravenous tobramycin and metronidazole as an adjunct to corticosteroids in acute, severe ulcerative colitis. Am J Gastroenterol 1994; 89 (1): 43-6.

25. Mantzaris GJ, Petraki K, Archavlis E, Amberiadis P, Kourtessas D, Christidou A, et al. A prospective randomized controlled trial of intravenous ciprofloxacin as an adjunct to corticosteroids in acute, severe ulcerative colitis. Scand J Gastroenterol 2001; 36 (9): 971-4. 
26. Demmler GJ. Cytomegalovirus infection: back to the future or no more elephants? Clin Infect Dis 2006; 43 (9): 1152-3.

27. Ho M. Epidemiology of cytomegalovirus infections. Rev Infect Dis 1990; 12 Suppl 7: S701-10.

28. Abarca K, Vial PA, Zamorano J, Paris C, Ferres M, Villarroel L, et al. [Seroprevalence of cytomegalovirus and Toxoplasma gondii in healthy subjects under 30 years old in Santiago, Chile]. Rev Med Chile 1997; 125 (5): 531-8.

29. Gandhi MK, Khanna R. Human cytomegalovirus: clinical aspects, immune regulation, and emerging treatments. Lancet Infect Dis 2004; 4 (12): 725-38.

30. Loewendorf A, Benedict CA. Modulation of host innate and adaptive immune defenses by cytomegalovirus: timing is everything. J Intern Med 2010; 267 (5): 483-501.

31. Pillet S, Pozzetto B, Jarlot C, Paul S, Roblin X. Management of cytomegalovirus infection in inflammatory bowel diseases. Dig Liver Dis 2012; 44 (7): 541-8.

32. Papadakis KA, Tung JK, Binder SW, Kam LY, Abreu MT, Targan SR, et al. Outcome of cytomegalovirus infections in patients with inflammatory bowel disease. Am J Gastroenterol 2001; 96 (7): 2137-42.

33. Baumgart DC, Sandborn WJ. Inflammatory bowel disease: clinical aspects and established and evolving therapies. Lancet 2007; 369 (9573): 1641-57.

34. Berk T, Gordon SJ, Choi HY, Cooper HS. Cytomegalovirus infection of the colon: a possible role in exacerbations of inflammatory bowel disease. Am J Gastroenterol 1985; 80 (5): 355-60.

35. Vega R, Bertrán X, Menacho M, Domenech E, Moreno de Vega V, Hombrados M, et al. Cytomegalovirus infection in patients with inflammatory bowel disease. Am J Gastroenterol 1999; 94 (4): 1053-6.

36. Cottone M, Pietrosi G, Martorana G, Casa A, Pecoraro G, Oliva L, et al. Prevalence of cytomegalovirus infection in severe refractory ulcerative and Crohn's colitis. Am J Gastroenterol 2001; 96 (3): 773-5.

37. Criscuoli V, Casa A, Orlando A, Pecoraro G, Oliva L, Traina M, et al. Severe acute colitis associated with CMV: a prevalence study. Dig Liver Dis 2004; 36 (12): 818-20.

38. Dimitroulia E, Spanakis N, Konstantinidou AE, Legakis NJ, Tsakris A. Frequent detection of cytomegalovirus in the intestine of patients with inflammatory bowel disease. Inflamm Bowel Dis 2006; 12 (9): 879-84.

39. Kuwabara A, Okamoto H, Suda T, Ajioka Y, Hatakeyama K. Clinicopathologic characteristics of clinically relevant cytomegalovirus infection in inflammatory bowel disease. J Gastroenterol 2007; 42 (10): 823-9.

40. Nakase H, Yoshino T, Honzawa Y, Chiba T. Low preva- lence of CMV infection in patients with Crohn's disease in comparison with ulcerative colitis: effect of different immune response on prevalence of CMV infection. Dig Dis Sci 2010; 55 (5): 1498-9.

41. Roblin X, Pillet S, Berthelot P, Del Tedesco E, Phelip JM, Chambonniere ML, et al. Prevalence of cytomegalovirus infection in steroid-refractory Crohn's disease. Inflamm Bowel Dis 2012; 18 (7): E1396-7.

42. Kim JJ, Simpson N, Klipfel N, Debose R, Barr N, Laine L. Cytomegalovirus infection in patients with active inflammatory bowel disease. Dig Dis Sci 2010; 55 (4): 1059-65.

43. Leveque N, Brixi-Benmansour H, Reig T, Renois F, Talmud D, Brodard V, et al. Low frequency of cytomegalovirus infection during exacerbations of inflammatory bowel diseases. J Med Virol 2010; 82 (10): 1694-700.

44. Sager K, Alam S, Bond A, Chinnappan L, Probert CS. Review article: cytomegalovirus and inflammatory bowel disease. Aliment Pharmacol Ther 2015; 41 (8): 725-33.

45. Criscuoli V, Rizzuto MR, Montalbano L, Gallo E, Cottone M. Natural history of cytomegalovirus infection in a series of patients diagnosed with moderate-severe ulcerative colitis. World J Gastroenterol 2011; 17 (5): 633-8.

46. Kandiel A, Lashner B. Cytomegalovirus colitis complicating inflammatory bowel disease. Am J Gastroenterol 2006; 101 (12): 2857-65.

47. de la Hoz RE, Stephens G, Sherlock C. Diagnosis and treatment approaches of CMV infections in adult patients. J Clin Virol 2002; 25 Suppl 2: S1-12.

48. Boivin G, Handfield J, Toma E, Murray G, Lalonde R, Tevere VJ, et al. Evaluation of the AMPLICOR cytomegalovirus test with specimens from human immunodeficiency virus-infected subjects. J Clin Microbiol 1998; 36 (9): 2509-13.

49. Yoshino T, Nakase H, Ueno S, Uza N, Inoue S, Mikami $S$, et al. Usefulness of quantitative real-time PCR assay for early detection of cytomegalovirus infection in patients with ulcerative colitis refractory to immunosuppressive therapies. Inflamm Bowel Dis 2007; 13 (12): 1516-21.

50. Shukla T, Singh S, Loftus EV, Jr., Bruining DH, McCurdy JD. Antiviral Therapy in Steroid-refractory Ulcerative Colitis with Cytomegalovirus: Systematic Review and Meta-analysis. Inflamm Bowel Dis 2015.

51. Kim YS, Kim YH, Kim JS, Cheon JH, Ye BD, Jung SA, et al. The prevalence and efficacy of ganciclovir on steroid-refractory ulcerative colitis with cytomegalovirus infection: a prospective multicenter study. J Clin Gastroenterol 2012; 46 (1): 51-6. 
52. Roblin X, Pillet S, Oussalah A, Berthelot P, Del Tedesco E, Phelip JM, et al. Cytomegalovirus load in inflamed intestinal tissue is predictive of resistance to immunosuppressive therapy in ulcerative colitis. Am J Gastroenterol 2011; 106 (11): 2001-8.

53. Maconi G, Lombardini M, Furfaro F, Bezzio C, Zerbi $\mathrm{P}$, Ardizzone S. Long-term outcome of inflammatory bowel diseases with cytomegalovirus colitis: effect of antiviral treatment. Eur J Gastroenterol Hepatol 2014; 26 (10): 1146-51.

54. Lennard-Jones JE, Ritchie JK, Hilder W, Spicer CC. Assessment of severity in colitis: a preliminary study. Gut 1975; 16 (8): 579-84.

55. Travis SP, Farrant JM, Ricketts C, Nolan DJ, Mortensen NM, Kettlewell MG, et al. Predicting outcome in severe ulcerative colitis. Gut 1996; 38 (6): 905-10.

56. Prantera C, Lorenzetti R, Cerro P, Davoli M, Brancato G, Fanucci A. The plain abdominal film accurately estimates extent of active ulcerative colitis. J Clin Gastroenterol 1991; 13 (2): 231-4.

57. Buckell NA, Williams GT, Bartram CI, Lennard-Jones JE. Depth of ulceration in acute colitis: correlation with outcome and clinical and radiologic features. Gastroenterology 1980; 79 (1): 19-25.

58. Carbonnel F, Lavergne A, Lemann M, Bitoun A, Valleur P, Hautefeuille P, et al. Colonoscopy of acute colitis. A safe and reliable tool for assessment of severity. Dig Dis Sci 1994; 39 (7): 1550-7.

59. Bitton A, Buie D, Enns R, Feagan BG, Jones JL, Marshall JK, et al. Treatment of hospitalized adult patients with severe ulcerative colitis: Toronto consensus statements. Am J Gastroenterol 2012; 107 (2): 179-94; author reply 95.

60. Lochs H, Dejong C, Hammarqvist F, Hebuterne X, Leon-Sanz M, Schutz T, et al. ESPEN Guidelines on Enteral Nutrition: Gastroenterology. Clin Nutr 2006; 25 (2): 260-74.

61. Alastair F, Emma G, Emma P. Nutrition in inflammatory bowel disease. JPEN J Parenter Enteral Nutr 2011; 35 (5): 571-80.

62. Valentini L, Schulzke JD. Mundane, yet challenging: the assessment of malnutrition in inflammatory bowel disease. Eur J Intern Med 2011; 22 (1): 13-5.

63. Klaassen J, Zapata R, Mella JG, Aguayo G, Alvarado D, Espinosa $\mathrm{O}$, et al. [Enteral nutrition in severe ulcerative colitis. Digestive tolerance and nutritional efficiency]. Rev Med Chile 1998; 126 (8): 899-904.

64. White JV, Guenter P, Jensen G, Malone A, Schofield M. Consensus statement: Academy of Nutrition and Dietetics and American Society for Parenteral and Enteral Nutrition: characteristics recommended for the identification and documentation of adult malnutrition (undernutrition). JPEN J Parenter Enteral Nutr 2012; 36 (3): 275-83.

65. Zachos M, Tondeur M, Griffiths AM. Enteral nutritional therapy for induction of remission in Crohn's disease. Cochrane Database Syst Rev 2007 (1): CD000542.

66. Wilson A, Reyes E, Ofman J. Prevalence and outcomes of anemia in inflammatory bowel disease: a systematic review of the literature. Am J Med 2004; 116 Suppl 7A: 44S-9S.

67. Truelove SC, Jewell DP. Intensive intravenous regimen for severe attacks of ulcerative colitis. Lancet 1974; 1 (7866): 1067-70.

68. Rosenberg W, Ireland A, Jewell DP. High-dose methylprednisolone in the treatment of active ulcerative colitis. J Clin Gastroenterol 1990; 12 (1): 40-1.

69. Bossa F, Fiorella S, Caruso N, Accadia L, Napolitano G, Valvano MR, et al. Continuous infusion versus bolus administration of steroids in severe attacks of ulcerative colitis: a randomized, double-blind trial. Am J Gastroenterol 2007; 102 (3): 601-8.

70. Laharie D, Bourreille A, Branche J, Allez M, Bouhnik Y, Filippi J, et al. Ciclosporin versus infliximab in patients with severe ulcerative colitis refractory to intravenous steroids: a parallel, open-label randomised controlled trial. Lancet 2012; 380 (9857): 1909-15.

71. Maser EA, Deconda D, Lichtiger S, Ullman T, Present $\mathrm{DH}$, Kornbluth A. Cyclosporine and infliximab as rescue therapy for each other in patients with steroid-refractory ulcerative colitis. Clin Gastroenterol Hepatol 2008; 6 (10): 1112-6.

72. Manosa M, López San Roman A, García-Planella E, Bastida G, Hinojosa J, González-Lama Y, et al. Infliximab rescue therapy after cyclosporin failure in steroid-refractory ulcerative colitis. Digestion 2009; 80 (1): 30-5.

73. Zúñiga A, Molina ME, García Huidobro I, López F, Rahmer A, Duarte I, et al. Colectomía total de urgencia por colitis ulcerosa grave. Rev Chil Cir 2004; 56 (1): 21-6.

74. Alves A, Panis Y, Bouhnik Y, Maylin V, Lavergne-Slove A, Valleur P. Subtotal colectomy for severe acute colitis: a 20-year experience of a tertiary care center with an aggressive and early surgical policy. J Am Coll Surg 2003; 197 (3): 379-85.

75. Hyman NH, Cataldo P, Osler T. Urgent subtotal colectomy for severe inflammatory bowel disease. Dis Colon Rectum 2005; 48 (1): 70-3.

76. Chung TP, Fleshman JW, Birnbaum EH, Hunt SR, Dietz DW, Read TE, et al. Laparoscopic vs open total abdominal colectomy for severe colitis: impact on recovery and subsequent completion restorative proctectomy. Dis Colon Rectum 2009; 52 (1): 4-10. 